International Journal of Child, Youth and Family Studies (2014) 5(1): 1-3

\title{
INTRODUCTION TO THE SPECIAL ISSUE ON YOUTH SUICIDE PREVENTION: RESEARCH, POLICY, AND PRACTICE
}

\author{
Jennifer White, Co-editor, and Patti Ranahan, Guest Co-editor
}

We are pleased to introduce this special issue of the International Journal of Child, Youth and Family Studies (IJCYFS) on youth suicide prevention. This issue brings together a diverse and exciting range of perspectives on the topic of youth suicide prevention. Original research, theoretical contributions, and critical reflections on practice are all represented here, underscoring the importance of multiple and collaborative approaches to addressing youth suicide, which is a leading cause of death among this age group.

We begin the issue with a series of groundbreaking original research articles on youth suicide prevention. First, Patti Ranahan examines child and youth care professionals' observational practices in the article, Watching in child and youth care suicide interventions: The potential for observation practices to be disengaging. In this grounded theory study, Ranahan illuminates the complexities and intricacies of the common practice of observation in situations where youth are suicidal. Then Chelsea Ohlmann, Janelle Kwee, and Robert Lees report the findings from their qualitative study, which emphasizes the importance of youth participation in suicide prevention efforts. Their article, Listening for the voices of resilience: A group of adolescents' experiences with a suicide prevention education program describes a novel methodology for capturing the voices of young people which has important implications for practice. Next, Kim Gryglewicz, Meredith Elzy, Renee Brown, Krista Kutash, and Marc Karver

Jennifer White, Ed.D., co-editor of the International Journal of Child, Youth and Family Studies, is an Associate Professor in the School of Child and Youth Care, University of Victoria, P.O. Box 1700, STN CSC, Victoria B.C., Canada, V8W 2 Y2. E-mail: jhwhite@uvic.ca

Patti Ranahan, Ph.D., guest co-editor of this Special Issue, is an Assistant Professor in the Department of Applied Human Sciences at Concordia University, 7141 Sherbrooke St. West, Montréal, Québec, Canada, H4B 1R6. E-mail: Patti.Ranahan@concordia.ca 
International Journal of Child, Youth and Family Studies (2014) 5(1): 1-3

present results from their community-based research project in their article, It's time to talk about it: Utilizing a community-based research approach to develop a family guide for youth suicide prevention. The authors developed a family guide as a resource to enhance awareness in youth suicide prevention. This is an area that has not received much attention to date and represents an important contribution to our field.

Another series of research articles focuses on specific groups, many of which experience disproportionately high rates of suicidal behaviour compared with the general population. In their article, Family and youth vulnerability to suicidal behavior in First Nations: A comparison of reserve and non-reserve groups, Michel Tousignant, Nathalie Morin, Livia Vitenti, Antoine Bibaud De Serres, and Arlene Laliberté examine the particular vulnerability of Aboriginal youth to suicide. Their findings suggest that prevention initiatives that include a focus on ecological factors and promote parental empowerment through family interventions are likely to hold promise. In the next article, School-based strategies to reduce suicidal ideation, suicide attempts, and discrimination among sexual minority and heterosexual adolescents in western Canada, youth in school are the primary focus. Elizabeth Saewyc, Chiaki Konishi, Hilary Rose, and Yuko Homma explore the relationship between the implementation of school-based programs and policies to prevent anti-homophobic bullying, and the experiences of lesbian, gay, bisexual, mostly heterosexual, and exclusively heterosexual young people. Results of their analysis of a province-wide survey in British Columbia suggest that school-level interventions, such as Gay Straight Alliances, may be an effective approach to reducing risks for suicide among sexual minority youth, while also offering prevention benefits for heterosexual boys. Shelley Craig, Lauren McInroy, Ramona Alaggia, and Lance McCready offer an in-depth critique of an online video project from the perspectives of sexual minority youth in their article, “Like picking up a seed, but you haven't planted it”: Queer youth analyze the It Gets Better project. Using a grounded theory approach, Craig and colleagues found that the It Gets Better project may raise awareness on bullying and suicide, however it has limitations for young people experiencing significant distress. We close our section on specific populations with a comprehensive literature review conducted by Anne Rhodes, Jennifer Bethell, and Lil Tonmyr entitled, Child sexual abuse and youth suicide: A review of the evidence with implications for future research. In this article the authors examine the evidence for potential sex differences in the association between child sexual abuse and suicide. They conclude that the relationship between child sexual abuse and youth suicide remains unclear and the potential sex differences in the association remain largely unaddressed.

The next set of articles focus on practice. First, Lyndsay Wells provides an historical account of the development and implementation of two youth suicide prevention programs offered within school settings, also in British Columbia, Canada. In her article, Connection and relational engagement in a youth suicide prevention program, Wells emphasizes the need to pay attention to the space that is created between students and practitioners in suicide education. Next, Kees Maas offers a practitioner's account of the implementation of a provincial suicidal prevention protocol in the 
Province of Quebec. His article, Suicide prevention in child welfare: The Quebec experience protecting children and staff explores the impact of standardizing approaches and defining interdependent levels and supports when intervening in situations where young people are suicidal. Finally, the importance of enhancing family wellness through community engagement with Aboriginal communities is explored in the article by Darien Thira. His article, Aboriginal youth suicide prevention: A post-colonial community-based approach describes the core elements of a youth suicide prevention program, which has been offered within Aboriginal communities over the past 20 years. The final article is a provocative theoretical piece by Scott Kouri and Jennifer White entitled, Thinking the other side of youth suicide: Engagements with life. This article invites us to consider "new spaces for thinking" and sets the stage for novel approaches to theorizing youth suicide and suicide prevention.

Collectively, all of the authors in this special issue showcase the value of bringing multiple perspectives to bear on this complex and challenging issue, thus extending our current knowledge of youth suicide prevention, practice, policy, and research. 